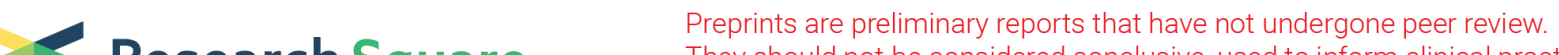 Research Square They should not be considered conclusive, used to inform clinical practice, or referenced by the media as validated information.
}

\section{Comparison of The Efficacy and Safety of Sedation Protocols With The Use of Dexmedetomidine- Remifentanil and Propofol-Remifentanil During Percutaneous Closure of Atrial Septal Defects}

\section{Xiao-Lan Chen}

Department of Intensive Care Unit, Affliated First Hospital of Fujian Medical University

Wen-Hui Huang

Anesthesiology Research Institute, the First Affiliated Hospital of Fujian Medical University

Yi-Han Zheng

Department of Cardiovascular Surgry, Fujian Medical University Union Hospital

Guican Zhang ( $\sim$ zhangguican123@163.com )

Fujian Medical University Union Hospital https://orcid.org/0000-0003-1695-7555

\section{Research article}

Keywords: atrial septal defects, sedation, dexmedetomidine, propofol, remifentanil

Posted Date: June 29th, 2021

DOl: https://doi.org/10.21203/rs.3.rs-642153/v1

License: (c) (1) This work is licensed under a Creative Commons Attribution 4.0 International License.

Read Full License 


\section{Abstract \\ Background}

The study was aimed to compare the efficacy and safety of different sedation protocols of dexmedetomidine-remifentanil and propofol-remifentanil for percutaneous closure of atrial septal defects (ASD) under transthoracic echocardiography (TTE) guidance.

\section{Material and Methods}

A total of 64 ASD patients scheduled for percutaneous closure under TTE guidance were randomly allocated into the dexmedetomidine-remifentanil (D-R) group $(n=32)$ and the propofol-remifentanil (P-R) group $(n=32)$ for sedation. The incidence of hemodynamic and respiratory adverse events, arterial blood gas analysis, induction and recovery time, pain score, infusion rate of remifentanil, satisfaction of the surgeon and patient, additional sedatives were collected for analysis and comparison.

\section{Results}

The induction time was longer in the D-R group than in the P-R group $(P<0.001)$. No differences were observed in the 2 groups in terms of the additional sedatives, infusion rate of remifentanil, pain score, recovery time $(P>0.05)$. There was no difference between the two groups regarding the incidence of hemodynamic instability $(P>0.05)$. Respiratory adverse events and hypercapnia $(P<0.05)$ were significantly less frequent in the D-R group.

\section{Conclusions}

Except for the induction time that was more rapid and the surgeon satisfaction score was higher in the propofol-remifentanil protocol, the efficacy was similar between two sedation protocols. The hemodynamic stability was comparable, the dexmedetomidine-remifentanil protocol had superior airway security since it was associated with fewer hypercapnia and respiratory adverse events.

\section{Background:}

Total transthoracic echocardiography (TTE), which is used to monitor and guide the procedure of percutaneous atrial septal defects (ASD) closure, is a suitable alternative method for avoiding exposure from X-ray radiation and esophageal probe $[1,2]$. While the spontaneous movements and agitation response to surgery, which might induce technical difficulties and failures of the procedure. Proper sedation and analgesia can alleviate discomfort and provide amnesia. ASD patients may experience prolonged onset time and delayed peak time of intravenous drugs due to the presence of a left-to-right shunt. It will lead to undesirable respiratory and cardiovascular depression if delivery of sedatives is not 
carefully titrated $[3,4]$. The procedure did not require transesophageal echocardiography. However, it is imperative to identify shallower yet equally effective sedation protocols associated with fewer sedativerelated adverse events for this specific cohort of patients.

It is difficult to control sedation depth with propofol. In addition to dose-dependent respiratory depression, propofol might not retain airway security especially given in combination with opioid agonists in nonintubated patients $[5,6]$. Conversely, dexmedetomidine does not decrease respiratory function when administered at appropriate doses. However, hemodynamic side effects associated with dexmedetomidine, including hypertension, hypotension and bradycardia, may limit its clinical application [7-9].

In this study, remifentanil combined with dexmedetomidine or propofol were all administered as a continuous intravenous infusion to be titrated carefully for achieving a proper sedation level. We hypothesized that there were more obvious hemodynamic changes while fewer respiratory adverse events in the dexmedetomidine-remifentanil protocol in comparison to the propofol-remifentanil protocol.

\section{Materials And Methods:}

This prospective, randomized and double-blind clinical research was approved by the Ethics Committee of Fujian Medical University, China (No. 2020KY018). Informed consent was obtained from all patients before the procedure. The study was registered at http:// www.chictr.org.cn (No. ChiCTR2000030969).

\section{Patients}

We included 64 ASD patients aged $\geq 18$ years old, American Society of Anesthesiologists (ASA) physical status $<$ IV and scheduled for percutaneous ASD device (Amplatzer atrial septal occluder) closure under TTE guidance from March 2020 to January 2021. Indications for the procedure included hemodynamically significant left to right shunts, a single secundum ASD without any other intracardiac structural abnormality, sufficient rims, satisfactory transthoracic acoustic windows. The exclusion criteria were as follows: STOP-BANG score $\geq 3$, severe renal, heart rate $<60$ beats per minute, second- or thirddegree block, hepatic, or heart failure, known drug allergies, or a history of drug abuse $[10,11]$.

The patients were randomly allocated into the dexmedetomidine-remifentanil (D-R) group $(n=32)$ or the propofol-remifentanil $(P-R)$ group $(n=32)$ for sedation by a random number table. Among the 64 patients, 2 patients were converted to surgical repair under general anesthesia and 3 patients with poor transthoracic acoustic windows were guided by transesophageal echocardiography (TEE), those patients were excluded from the study. Finally, data for 59 patients (29 patients in the D-R group and 30 patients in the P-R group) were analyzed.

\section{Sedation Protocols}

Both the surgeons and patients were blinded to the sedation protocol. None of the patients were premedicated. The sedation level was assessed with the Observer's Assessment of Alertness/Sedation 
(OAA/S) scale [12] and bispectral index score (BIS, Aspect Medical System, Newton, MA, USA). Intraoperative sedation levels were targeted to achieve a BIS of $60-85$ and an OAA/S score $\leq 4$. Remifentanil (Ultiva ${ }^{\circledR}$, China National Pharmaceutical Industry Corporation Ltd., HeBei, China) was infused continuously at a rate of $4 \mu \mathrm{g} / \mathrm{kg}$ per hour in both groups before starting the procedure. For the $D-$ $R$ group, the initial infusion of dexmedetomidine (Precedex®, Yangtze River Pharmaceutical (Group) Co. Ltd., JiangSu, China) was set at $6.0 \mu \mathrm{g} / \mathrm{kg} / \mathrm{h}$ for 10 minutes as the initial loading dose, followed by a maintenance infusion beginning at a rate of $1.0 \mu \mathrm{g} / \mathrm{kg} / \mathrm{h}$. In the P-R group, the initial infusion of propofol (Pofol ${ }^{\circledR}$, B.Braun Melsungen AG, Melsungen, Germany) was set at $6.0 \mathrm{mg} / \mathrm{kg} / \mathrm{h}$ for 10 minutes, followed by continuous infusion beginning at a rate of $2 \mathrm{mg} / \mathrm{kg} / \mathrm{h}$. When target sedation level was obtained, the maintenance infusion rate of sedatives was adjusted according to the patients' sedation level and all drugs were discontinued at the end of the procedure in both groups.

If sedation level was inadequate in either group, the infusion rates of sedatives were increased at first. Besides, a bolus of 10-20 mg propofol was administered as a rescue sedation therapy when first-line treatment failed [13].

\section{Anesthesia management and data collection}

Vital signs were monitored continuously and recorded at 5-min intervals: oxygen saturation $\left(\mathrm{SpO}_{2}\right)$, heart rate (HR), electrocardiogram (ECG), respiratory rate (RR). In addition, an arterial catheter was routinely inserted for assessing invasive arterial pressure, and arterial blood gas analysis was performed at baseline (breathing room air). All patients were breathing spontaneously, and $4 \mathrm{~L} /$ min oxygen was given through a nasal cannula. The sedation protocols were started after placement of the arterial catheter in both groups. On achieving the targeted sedation level (BIS of $60-85$ and an OAA/S score $\leq 4$ ), the right groin was infiltrated with $10 \mathrm{~mL}$ of $1 \%$ lidocaine at the beginning of the interventional procedure.

Arterial blood gas analysis was repeated immediately after the procedure. The number of patients with hypercapnia $\left(\mathrm{PaCO}_{2} \geq 45 \mathrm{mmHg}\right)$ was evaluated. The time needed to achieve an Aldrete score $\geq 9$ weas noted [14]. Patients were asked to evaluate their levels of pain $(0=$ no; $1-3=$ mild; $4-6=$ moderate; $7-10$ = severe pain) by using the visual analogue scale (VAS) and were transferred to the ward when the Aldrete score was $\geq 9$ [3]. The satisfaction with the quality of the sedation (5-point Likert scale: 1, very satisfied; 2 , satisfied; 3 , neutral; 4, dissatisfied; and 5, very dissatisfied) were evaluated by the surgeons and patients [11].

Hemodynamic and respiratory adverse events were defined as follows. Hypotension (mean arterial blood pressure $<65 \mathrm{mmHg}$ ), hypertension (mean arterial blood pressure $\geq 20 \%$ of baseline), bradycardia (heart rate $<50$ beats per minute), bradypnea (respiratory rate $<8 / \min$ for $\geq 1$ minute), apnea (absence of ventilator effort $\geq 20$ seconds) and oxygen desaturation (SpO2 $<90 \%$ for $\geq 10$ seconds) were all recorded [11].

We managed adverse respiratory events with a jaw thrust, mask ventilation, by increasing oxygen flow, or asopharyngeal/ oropharyngeal airway insertion. Noradrenaline, urapidil, atropine, or esmolol was 
administered for adverse hemodynamic events.

The efficacy of sedation protocols was assessed on the ability to successfully complete the procedure without rescue sedatives, pain score, infusion rate of remifentanil, induction time (the time to achieve targeted levels of sedation), recovery time (the time to an Aldrete score $\geq 9$ ), anesthetic satisfaction of the surgeons and patients. The incidence of hemodynamic and respiratory adverse events, arterial blood gas analysis, hypercapnia all were compared to evaluate the safety of sedation protocols.

\section{Interventional Procedure and Echocardiography Guidance}

The patient was placed in a supine position, and TTE was performed continuously throughout the procedure to monitor device deployment. The interventional procedure of percutaneous closure of ASD has been described in the previous study [1]. Briefly, a venous sheath was inserted after the right femoral vein was punctured and systemic heparinization $(1 \mathrm{mg} / \mathrm{kg}$ ) was performed. Subsequently, a catheter was inserted, followed by advancement of a guidewire through the ASD into the left atrium. Then, an occluder was delivered carefully through the sheath. The left disc was deployed in the left atrium and pulled back against the atrial septum, and then the right atrial disc was deployed. Finally, the device was released once the occluder was positioned properly.

If patients did not undergo successful device implantation due to severe residual shunt, complete heart block, or device dislodged, then the patients were referred for surgical repair under general anesthesia with endotracheal intubation as a remedial measure and were excluded from the final sedation analysis.

\section{Statistical Analysis}

Statistical analyses were performed by SPSS software (ver.22.0, SPSS Inc., Chicago, IL, United States). The results are presented as the mean \pm standard deviation (SD) for the continuous variables and as the numbers or percentages for categorical variables. Continuous and categorical variables were tested by using Student's t test and $\chi^{2}$ test or Fisher's exact test, respectively. For comparison of the grade data, Wilcoxon rank- sum test was performed. All statistical tests were two-tailed with a significance level of 0.05 .

The main safety concern with sedation in patients is respiratory adverse events. According to a previous study, propofol-remifentanil combinations for sedation during hysteroscopy, the incidence of respiratory depression was $40 \%$. Based on this result, the decreases in the incidence of respiratory depression to $10 \%$ in dexmedetomidine-remifentanil group was considered clinically significant [15]. A sample size of 32 subjects per group was required considering a one-sided test with $a=0.025$, power of $80 \%(\beta=0.20)$ and assuming a $10 \%$ drop-out rate.

\section{Results:}

The demographic and clinical characteristics are presented in Table 1. There were no significant differences between the two groups $(P>0.05)$. 
Table 1

The data of demographic and clinical characteristics

\begin{tabular}{|c|c|c|c|c|}
\hline & D-R group $(n=29)$ & $\begin{array}{l}\text { P-R group } \\
(n=30)\end{array}$ & tor $\chi 2$ value & $P$ value \\
\hline Age $(y)$ & $45.34 \pm 15.80$ & $43.53 \pm 16.31$ & 0.433 & 0.667 \\
\hline Women, n (\%) & $20(69.0 \%)$ & $24(80.0 \%)$ & 0.947 & 0.330 \\
\hline Height (cm) & $162.03 \pm 8.34$ & $160.03 \pm 8.05$ & 0.685 & 0.352 \\
\hline Weight (kg) & $57.02 \pm 10.06$ & $57.88 \pm 11.06$ & -0.314 & 0.754 \\
\hline BMI (kg/m2) & $21.62 \pm 2.91$ & $22.37 \pm 3.19$ & -0.938 & 0.352 \\
\hline ASA status (II/III), n & $22 / 7$ & $24 / 6$ & 0.147 & 0.701 \\
\hline Snoring history, n (\%) & $8(27.6 \%)$ & 10(33.3\%) & 0.230 & 0.632 \\
\hline Smoking history, n (\%) & $7(24.1 \%)$ & $5(16.7 \%)$ & 0.508 & 0.476 \\
\hline ASD size $(\mathrm{mm})$ & $18.96 \pm 7.51$ & $17.77 \pm 6.88$ & 0.633 & 0.529 \\
\hline PASP (mm Hg) & $41.48 \pm 9.04$ & $39.03 \pm 16.31$ & 0.716 & 0.477 \\
\hline Qp/Qs & $2.27 \pm 0.32$ & $2.18 \pm 0.26$ & 1.154 & 0.253 \\
\hline
\end{tabular}

In terms of sedation efficacy (Table 2), all patients successfully completed procedure without apparent body movement leading to interruption of procedure. The induction time was longer in the $D-R$ group than in the P-R group $(P<0.001)$. Four patients $(13.8 \%)$ in the $\mathrm{D}-\mathrm{R}$ group and 2 patients $(6.7 \%)$ in the $\mathrm{P}-\mathrm{R}$ group required additional propofol administrated as a rescue sedative $(P=0.424)$ at doses of $17.50 \pm 5.00 \mathrm{mg}$ and $15.00 \pm 7.07 \mathrm{mg}(P=0.633)$, respectively. The infusion rates of remifentanil and maximal pain scores (VAS) were not significantly different in the two groups $(P>0.05)$. No difference between the D-R group and the P-R group was observed regarding the recovery time $(P=0.116)$. All patients were transferred to the ward within 20 minutes after surgery. The patient satisfaction score was comparable $(P=0.668)$, whereas the surgeon satisfaction score was higher in P-R group than in D-R group $(P=0.006)$. 
Table 2

Efficacy of sedation protocols in both groups.

\begin{tabular}{|c|c|c|c|c|}
\hline & $\begin{array}{l}\text { D-R group }(n= \\
29)\end{array}$ & $\begin{array}{l}\text { P-R group } \\
(n=30)\end{array}$ & $\begin{array}{l}t \text { or } \chi 2 \text { or } Z \\
\text { value }\end{array}$ & $\begin{array}{l}P \\
\text { value }\end{array}$ \\
\hline $\begin{array}{l}\text { Induction time } \\
\text { (min) }\end{array}$ & $17.66 \pm 2.65$ & $\begin{array}{l}11.43 \pm \\
1.48\end{array}$ & 11.088 & $\begin{array}{l}< \\
0.001\end{array}$ \\
\hline Procedure duration (min) & $52.48 \pm 9.11$ & $\begin{array}{l}52.23 \pm \\
7.41\end{array}$ & 0.116 & 0.908 \\
\hline Sedation duration (min) & $70.14 \pm 9.62$ & $\begin{array}{l}63.67 \pm \\
7.02\end{array}$ & 2.959 & 0.004 \\
\hline $\begin{array}{l}\text { Remifentanil infusion rate }(\mu \mathrm{g} \cdot \mathrm{kg}- \\
1 \cdot \mathrm{h}-1)\end{array}$ & $3.99 \pm 0.08$ & $3.97 \pm 0.09$ & 0.709 & 0.481 \\
\hline Maximum pain level (VAS) & & & 0.074 & 0.786 \\
\hline 0 (no pain) & $24(82.8 \%)$ & $24(80.0 \%)$ & & \\
\hline$\varangle 3$ (mild pain) & $5(17.2 \%)$ & $6(20.0 \%)$ & & \\
\hline Recovery time (min) & $13.03 \pm 1.82$ & $\begin{array}{l}12.20 \pm \\
2.17\end{array}$ & 1.596 & 0.116 \\
\hline \multicolumn{5}{|l|}{ Additional propofol required } \\
\hline Patients, n (\%) & $4(13.8 \%)$ & $2(6.7 \%)$ & / & 0.424 \\
\hline Dose (mg) & $17.50 \pm 5.00$ & $\begin{array}{l}15.00 \pm \\
7.07\end{array}$ & 0.516 & 0.633 \\
\hline Surgeon satisfaction score & & & -2.722 & 0.006 \\
\hline 1 (very satisfied) & $11(37.9 \%)$ & $22(73.3 \%)$ & & \\
\hline 2 (satisfied) & $12(41.4 \%)$ & $6(20.0 \%)$ & & \\
\hline 3 (neutral) & $6(20.7 \%)$ & $2(6.7 \%)$ & & \\
\hline Patient satisfaction score & & & -0.429 & 0.668 \\
\hline 1 (very satisfied) & $23(79.3 \%)$ & $22(73.3 \%)$ & & \\
\hline 2 (satisfied) & $4(13.8 \%)$ & $7(23.3 \%)$ & & \\
\hline 3 (neutral) & $2(6.9 \%)$ & $1(3.3 \%)$ & & \\
\hline
\end{tabular}

Table 3 shows the incidence of intraoperative adverse events in both groups. In terms of sedation safety, we did not find difference on incidence of cardiovascular adverse events between the two groups $(P=$ 
0.506). Two patients in D-R group developed transient hypertension and did not require therapy. The incidence of adverse respiratory events in the $D-R$ group was significantly lower than that in the P-R group ( $3.4 \%$ vs $26.7 \%$, respectively; $P=0.026)$. All adverse respiratory events in the two groups were mild in severity; the insertion of asopharyngeal/ oropharyngeal airway was not required in either group.

Table 3

The incidence of intraoperative adverse events in both groups.

\begin{tabular}{|c|c|c|c|c|}
\hline & $\begin{array}{l}\text { D-R group } \\
(n=29)\end{array}$ & $\begin{array}{l}\text { P-R group } \\
(n=30)\end{array}$ & $\chi 2$ value & $P$ value \\
\hline Respiratory adverse events, $\mathrm{n}(\%)$ & $1(3.4 \%)$ & $8(26.7 \%)$ & / & 0.026 \\
\hline Cardiovascular adverse events, n (\%) & $6(20.7 \%)$ & $4(13.3 \%)$ & / & 0.506 \\
\hline Hypertension, n (\%) & $2(6.9 \%)$ & $0(0 \%)$ & / & 0.237 \\
\hline Hypotension, n (\%) & $2(6.9 \%)$ & $3(10 \%)$ & / & 1.000 \\
\hline Bradycardia, n (\%) & $2(6.9 \%)$ & $1(3.3 \%)$ & / & 0.612 \\
\hline
\end{tabular}

Table 4 shows the outcome of the arterial blood gas analysis in both groups. Partial pressure of carbon dioxide $\left(\mathrm{PaCO}_{2}\right)$ values was significantly higher $(P=0.013)$ and hypercapnia was more frequent $(P=$ 0.012 ) in the P-R group than in the D-R group at the end of the procedure. 
Table 4

The outcome of the arterial blood gas analysis in both groups

\begin{tabular}{|c|c|c|c|c|}
\hline & $\begin{array}{l}\text { D-R group } \\
(n=29)\end{array}$ & $\begin{array}{l}\text { P-R group } \\
(n=30)\end{array}$ & $t$ or $\chi 2$ value & $P$ value \\
\hline \multicolumn{5}{|l|}{ Baseline } \\
\hline $\mathrm{pH}$ & $7.42 \pm 0.02$ & $7.42 \pm 0.03$ & -0.357 & 0.722 \\
\hline $\mathrm{PaO} 2(\mathrm{mmHg})$ & $79.21 \pm 4.43$ & $80.33 \pm 6.11$ & -0.809 & 0.422 \\
\hline $\mathrm{PaCO} 2(\mathrm{mmHg})$ & $35.83 \pm 3.30$ & $35.70 \pm 4.24$ & 0.129 & 0.898 \\
\hline \multicolumn{5}{|c|}{ At the end of the procedure } \\
\hline $\mathrm{PH}$ & $7.36 \pm 0.02$ & $7.34 \pm 0.02$ & 3.048 & 0.003 \\
\hline $\mathrm{PaO} 2(\mathrm{mmHg})$ & $130.03 \pm 4.99$ & $125.70 \pm 7.01$ & 2.728 & 0.008 \\
\hline $\mathrm{PaCO} 2(\mathrm{mmHg})$ & $41.52 \pm 4.22$ & $44.70 \pm 5.31$ & -2.554 & 0.013 \\
\hline $\begin{array}{l}\text { Hypercapnia, } \\
\text { n (\%) }\end{array}$ & $4(13.8 \%)$ & $13(43.3 \%)$ & 6.273 & 0.012 \\
\hline
\end{tabular}

\section{Discussion:}

This study has certain clinical implications for the sedation protocol of percutaneous ASD closure under TTE guidance. Patient tolerance is important for successful completion of a safe procedure. The technique of sedation combining an opioid as an analgesic is actually required for alleviating stimulation of local anesthetic injection, operative manipulation and continuous contact with the probe that may be pressed as necessary to obtain good images. However, the use of sedatives in combination with analgesics is usually associated with intraoperative hemodynamics and/or respiratory complications without manipulation of the airway $[16,17]$. Therefore, it is necessary to find an appropriate sedative regimen that not only improves patient comfort during the operation but also is associated with fewer sedative-related adverse events.

Combination of remifentanil was administered as an analgesic requirement for the insufficient local anesthetic infiltration to alleviate motor reaction to painful manipulation of the delivery sheath. Moreover, when considering that patient body movement under sedation is due to pain, simply deepening the level of sedation may not be the solution [16]. Therefore, we believed that continuous infusion of remifentanil enabled good tolerance of this procedure and avoided the use of excessive sedatives. Although the infusion rates of remifentanil and the procedure time were similar in both groups, the time of remifentanil 
infusion was longer in the D-R group than in the P-R group because of the longer induction time. Remifentanil hydrochloride is an ultra-short-acting opioid with rapid distribution and elimination processes, and the blood concentration typically decreases by 50\% 3-6 minutes after cessation of continuous infusion, regardless of its duration [11]. In our study, the infusion time of remifentanil would not prolong the recovery time as no difference of the value between the two groups was observed.

The main safety concern with sedation in patients is respiratory adverse events, which are mainly related to the use of sedatives and analgesics [16]. Many studies have reported that dexmedetomidine alone may not cause a decrease in respiratory or hypoventilation rates due to the central effect on respiration, but one must be vigilant with remifentanil $[10,11,16]$. Remifentanil is generally associated with respiratory depression, and this may result in airway obstruction due to relaxation of the pharyngeal muscles. Our study found that the incidence of adverse respiratory events in the D-R group was significantly lower than that in the P-R group. Then, we measured the $\mathrm{PaCO}_{2}$ level through arterial blood gas analysis, and an important finding in this study was the lower incidence of hypercapnia in the D-R group compared with the P-R group. Thus, the study further confirmed that dexmedetomidine in combination with remifentanil did not increase the risk of opioid-related respiratory depression, similar to the results of previous study $[18,19]$.

Given the predictable effect in cardiovascular system, we hypothesized that there were more obvious hemodynamic changes in the dexmedetomidine-remifentanil protocol than the propofol-remifentanil protocol. Some studies have found that a dose-dependent decrease in heart rate is the most common cardiovascular effect in patients receiving dexmedetomidine, while the rate is generally not lower than 50 beats/minute, and usually, anticholinergic drugs are not required to increase the heart rate [20, 21]. Recent studies have suggested that maintaining intraoperative MABP $\geq 65 \mathrm{mmHg}$ has equal clinical implications as conventional maintenance within $20 \%$ of preoperative baseline values $[20,22]$. In addition, we believe that due to the fear and anxiety of the impending procedure, preoperative baseline blood pressure may have higher initial levels before the use of sedatives. Once sedation begins, the blood pressure is probably below $20 \%$ of the baseline value during the procedure, which makes it inaccurate for assessment of hemodynamic safety. Based on these theories, we defined hypotension as an MABP $<65$ $\mathrm{mmHg}$ to assess hemodynamic instability in this study. Rather than large or rapid bolus injection during a short period of time, continuous infusion of dexmedetomidine for an initial loading dose was helpful to prevent the hemodynamic changes that are usually associated with dexmedetomidine and for maintaining stable anesthesia [23]. Finally, there was an infrequent and comparable incidence of cardiovascular adverse events in both groups, which differed from the initial hypothesis of hemodynamic changes in the dexmedetomidine-remifentanil protocol.

The primary efficacy end-point was the percentage of patients not requiring rescue sedatives based on achieving targeted sedation depth. Our study has already demonstrated that sedation protocols of both dexmedetomidine-remifentanil and propofol-remifentanil combinations have comparable efficacy for providing sufficient sedative and analgesic effects. However, physicians favored the propofolremifentanil-based sedation protocol perhaps due to the shorter induction time. Although the incidence of 
cardiovascular adverse events was comparable and that such events were easily managed in both groups, the dexmedetomidine- remifentanil protocol had superior airway security since it is associated with fewer hypercapnia and respiratory adverse events than the propofol-remifentanil protocol. The best approach to sedation for patients in percutaneous closure of ASD is to choose a sedation regimen tailored according to the clinical risk assessment. For those patients with the uncertainties in airway safety, dexmedetomidine-remifentanil-based sedation protocol may be a suitable sedation approach.

Intracardiac shunts can change the onset time of sedation in ASD patients [23]. Previous study demonstrated that significantly delayed pharmacodynamics responses to neuromuscular blocking agent cisatracurium were observed in patients with septal defects [24]. In this study, there was a longer time needed for induction to achieve targeted levels of sedation with dexmedetomidine than with propofol, a result that is also similar to the results of previous research above the patients without intracardiac shunts [10]. However, there is no clinical study involving adequate number of patients has been done to explore the magnitude of difference in the induction time of anesthesia in patients with or without intracardiac shunt $[25,26]$. Simple dosage regimens based only on patient weight may not result in stable effect-site concentrations because of the complex pharmacokinetics and pharmacodynamics of each drug. Further exploration of pharmacokinetics and pharmacodynamics of sedatives in patients with intracardiac shunts assumed great significance in the formulation of a population-specific dosage regimen to achieve optimized therapy.

\section{Conclusions:}

In the term of efficacy, both sedation protocols have comparable efficacy for providing sufficient sedative and analgesic effects, however, the time to target sedation level was more rapid and the surgeon satisfaction score was higher in the propofol-remifentanil protocol than in the dexmedetomidineremifentanil sedation protocol. In terms of sedation safety, considering that the hemodynamic stability was comparable, we concluded that dexmedetomidine-remifentanil protocol had superior airway security since it was associated with fewer hypercapnia and respiratory adverse events.

\section{List Of Abbreviations}

ASD, atrial septal defects; TTE, transthoracic echocardiography; TEE, transesophageal echocardiography; D-R group, dexmedetomidine-remifentanil group; P-R group, propofol-remifentanil group; ASA, American Society of Anesthesiologists;

OAA/S, Observer's Assessment of Alertness/Sedation; BIS, bispectral index score;

VAS, visual analog scale; $\mathrm{PH}$, pondus hydrogenii; $\mathrm{PaO} 2$, partial pressure of oxygen; $\mathrm{PaCO} 2$, partial pressure of carbon dioxide.

\section{Declarations}


Ethics approval and consent to participate

The study was approved by the Ethics Committee of Fujian Medical University, China (No. 2020KY018) and was registered at http:// www.chictr.org.cn (No. ChiCTR2000030969), 20 March 2020.

Consent for publication

Informed consent for publication was obtained.

\section{Availability of data and materials}

Data used for this study are available upon request.

\section{Funding}

None.

\section{Data Availability Statement}

All relevant data are within the manuscript and its Supporting Information files.

Authors' contributions: XL C WH H YHZ GCZ

Conceptualization: XL C, GC Z.

Data curation: XL C, YH Z, WH H.

Formal analysis: $\mathrm{XL} \mathrm{C,} \mathrm{YH} \mathrm{Z,} \mathrm{WH} \mathrm{H.}$

Investigation: XL C, WH H.

Resources: XL C, WH H.

Software: XL C, YH Z, WH H.

Supervision: XL C, GC Z.

Validation: XL C, GC Z.

Visualization: XL C, WH H.

Writing-original draft: $\mathrm{XL} \mathrm{C}, \mathrm{WH} H$.

\section{Acknowledgements}

We would like to thank Zhao-Nan Zeng for help with the statistical analysis and the cardiac surgery team for their help and support in the management of these patients. 


\section{Competing interests}

The authors have nothing to declare with regards to this project.

\section{References}

1. Ding C, Chang JK, Lin CC, et al. Efficacy and Safety of Transthoracic Echocardiography Alone in Transcatheter Closure of Secundum-Type Atrial Septal Defects in Adults. Echocardiography. 2016;33(4):579-85.

2. Cao H, Chen Q, Zhang GC, et al. Percutaneous device closure of atrial septal defect with totally transthoracic echocardiography guide, without x-ray machine. Med (Baltim). 2016;95(44):e5256.

3. Koruk S, Mizrak A, Kaya UB, et al. Propofol/dexmedetomidine and propofol/ketamine combinations for anesthesia in pediatric patients undergoing transcatheter atrial septal defect closure: a prospective randomized study. Clin Ther. 2010;32(4):701-9.

4. Gozal D, Rein AJ, Nir A, et al. Propofol does not modify the hemodynamic status of children with intracardiac shunts undergoing cardiac catheterization. Pediatr Cardiol. 2001;22(6):488-90.

5. Akin A, Guler G, Esmaoglu A, et al. A comparison of fentanyl-propofol with a ketamine-propofol combination for sedation during endometrial biopsy. J Clin Anesth. 2005;17(3):187-90.

6. Curtis JA, Hollinger MK, Jain HB. Propofol-based versus dexmedetomidine-based sedation in cardiac surgery patients. J Cardiothorac Vasc Anesth. 2013;27(6):1289-94.

7. Venn RM, Hell J, Grounds RM. Respiratory effects of dexmedetomidine in the surgical patient requiring intensive care. Crit Care. 2000;4(5):302-8.

8. Candiotti KA, Bergese SD, Bokesch PM, et al. MAC Study Group. Monitored anesthesia care with dexmedetomidine: a prospective, randomized, double-blind, multicenter trial. Anesth Analg. 2010;110(1):47-56.

9. Bhana N, Goa KL, McClellan KJ. Dexmedetomidine Drugs. 2000;59(2):263-70.

10. Arain SR, Ebert TJ. The efficacy, side effects, and recovery characteristics of dexmedetomidine versus propofol when used for intraoperative sedation. Anesth Analg. 2002;95(2):461-6.

11. St -PP, Tanoubi I, Verdonck O, et al. Dexmedetomidine Versus Remifentanil for Monitored Anesthesia Care During Endobronchial Ultrasound-Guided Transbronchial Needle Aspiration: A Randomized Controlled Trial. Anesth Analg. 2019;128(1):98-106.

12. Chernik DA, Gillings D, Laine $H$, et al. Validity and reliability of the Observer's Assessment of Alertness/Sedation Scale: study with intravenous midazolam. J Clin Psychopharmacol. 1990;10(4):244-51.

13. Kim N, Yoo YC, Lee SK, et al. Comparison of the efficacy and safety of sedation between dexmedetomidine-remifentanil and propofol-remifentanil during endoscopic submucosal dissection. World J Gastroenterol. 2015;21(12):3671-8.

14. Aldrete JA, Kroulik D. A postanesthetic recovery score. Anesth Analg. 1970;49(6):924-34. 
15. Park S, Choi SL, Nahm FS, et al. Dexmedetomidine-remifentanil vs propofol-remifentanil for monitored anesthesia care during hysteroscopy: Randomized, single-blind, controlled trial. Med (Baltim). 2020;99(43):e22712.

16. Goettel N, Bharadwaj S, Venkatraghavan L, et al. Dexmedetomidine vs propofol-remifentanil conscious sedation for awake craniotomy: a prospective randomized controlled trial. $\mathrm{Br} \mathrm{J}$ Anaesth. 2016;116(6):811-21.

17. Skucas AP, Artru AA. Anesthetic complications of awake craniotomies for epilepsy surgery. Anesth Analg. 2006;102(3):882-7.

18. Ge DJ, Qi B, Tang G, et al. Intraoperative Dexmedetomidine Promotes Postoperative Analgesia and Recovery in Patients after Abdominal Colectomy: A CONSORT-Prospective, Randomized, Controlled Clinical Trial. Med (Baltim). 2015;94(43):e1727.

19. Mayr NP, Wiesner G, van der Starre P, et al. Dexmedetomidine versus propofol-opioid for sedation in transcatheter aortic valve implantation patients: a retrospective analysis of periprocedural gas exchange and hemodynamic support. Can J Anaesth. 2018;65(6):647-57.

20. Edokpolo LU, Mastriano DJ, Serafin J, et al. Discharge Readiness after Propofol with or without Dexmedetomidine for Colonoscopy: A Randomized Controlled Trial. Anesthesiology. 2019;131(2):279-86.

21. Wang HM, Shi XY, Qin XR, et al. Comparison of dexmedetomidine and propofol for conscious sedation in inguinal hernia repair: A prospective, randomized, controlled trial. J Int Med Res. 2017;45(2):533-9.

22. Salmasi V, Maheshwari K, Yang DS, et al. Relationship between Intraoperative Hypotension, Defined by Either Reduction from Baseline or Absolute Thresholds, and Acute Kidney and Myocardial Injury after Noncardiac Surgery: A Retrospective Cohort Analysis. Anesthesiology. 2017;126(1):47-65.

23. Tobias JD, Gupta P, Naguib A, et al. Dexmedetomidine: applications for the pediatric patient with congenital heart disease. Pediatr Cardiol. 2011;32(8):1075-87.

24. Wu ZF, Wang S, Peng XM, et al. Altered Cisatracurium Pharmacokinetics and Pharmacodynamics in Patients with Congenital Heart Defects. Drug Metab Dispos. 2016;44(1):75-82.

25. Hasija S, Chauhan S, Jain P, et al. Comparison of speed of inhalational induction in children with and without congenital heart disease. Ann Card Anaesth. 2016;19(3):468-74.

26. Lipiec P, Miśkowiec D, Peruga JZ, et al. Conscious sedation for transcatheter implantation of atrial septal occluders with two- and three-dimensional transoesophageal echocardiography guidance - a feasibility and safety study. Kardiol Pol. 2018;76(2):406-12. 